\title{
Formación de grafito chunky en piezas de pequeño espesor fabricadas utilizando fundición de hierro con grafito esferoidal( ${ }^{\circ}$
}

\author{
I. Asenjo*, P. Larrañaga* y J. Sertucha*
}

\begin{abstract}
Resumen
El grafito chunky es una malformación detectada habitualmente en las zonas de última solidificación pertenecientes a piezas de fundición grafítica esferoidal con enfriamientos muy lentos. Estudios previos realizados sobre bloques cúbicos de 300 y $180 \mathrm{~mm}$ de lado y fabricados con aleaciones de matriz ferrítica, indican que los factores que promueven la formación de este defecto son: bajas velocidades de enfriamiento, excesiva post-inoculación del metal de colada y la presencia de elevados contenidos de silicio y/o cerio. La intensificación de estos factores en la fabricación de piezas con espesores más reducidos ha permitido obtener grafito chunky en secciones inferiores a $50 \mathrm{~mm}$. Se han utilizado diferentes condiciones experimentales y se han determinado los factores de mayor influencia en la formación del defecto. La utilización de técnicas analíticas de gran precisión ha mostrado una gran similitud en las composiciones químicas entre las zonas afectadas por la malformación grafítica y aquellas en las que el grafito aparece únicamente bajo formas perfectamente esferoidales. Por otra parte, los contenidos de oxígeno medidos en los materiales estudiados en este trabajo no muestran relación con la cantidad de grafito chunky formado, sin embargo sí dependen del tipo de elemento químico utilizado para realizar el tratamiento de esferoidización del metal fundido.
\end{abstract}

\section{Chunky graphite formation in small section ductile iron castings}

\begin{abstract}
Chunky graphite is a degenerated graphite form which can be found in the thermal centre of ductile iron heavy section castings. Previous studies made on cubic blocks ( 300 and $180 \mathrm{~mm}$ in side) manufactured using alloys with fully ferritic matrix structures show that low cooling rates, excessive post-inoculation and high silicon and/or cerium contents in the melts are the most important factors that promote this kind of defect. The enhancement of these critical factors led to obtain chunky graphite in sections lower than $50 \mathrm{~mm}$. Different experimental conditions have been used in order to establish the main parameters that affect this graphite malformation. The use of cutting-edge techniques in the analysis of chemical compositions has revealed that no significant differences can be found when comparing chunky areas and well-formed spheroidal graphite areas. On the other hand, it has not been possible to establish any correlation between the oxygen contents and the scale of the defect. However, it is noteworthy that the oxygen content is related to the use of magnesium or cerium as nodulizer agent.
\end{abstract}

Keywords Chunky graphite; Ductile iron; Small section casting; Cerium; Magnesium; Solidification curves.

\section{INTRODUCCIÓN}

El grafito chunky (GCH) es una degeneración grafítica constituida por una estructura ramificada e interconectada de grafito, habitualmente asociada a los centros térmicos de piezas de fundición grafítica esferoidal con elevado espesor ${ }^{[1-5]}$. La aparición de este defecto se relaciona con un efecto negativo sobre las propiedades mecánicas del material, por lo que su presencia en piezas con importantes requerimien- tos constituye un serio problema para la industria eólica y/o de automoción ${ }^{[3 \text { y } 6]}$. Aunque la formación del GCH ha sido objeto de un importante número de estudios con el fin de determinar cuáles son sus causas $^{[1-5}$ y $\left.7-9\right]$, aún hoy éstas no se conocen bien al igual que su mecanismo de formación y crecimiento. Un ejemplo de la controversia existente lo constituye el efecto de la inoculación del metal de colada sobre la formación de este tipo de malformación grafítica $^{[4-5}$ y 9].

(•) Trabajo recibido el día 25 de enero de 2011 y aceptado en su forma final el día 25 de mayo de 2011.

* Ingeniería y Procesos de Fundición. Centro de Investigación Metalúrgica AZTERLAN. Aliendalde Auzunea nº 6, E-48200 Durango

(Bizkaia), E-mail: jsertucha@azterlan.es 
Es necesario destacar que, salvo en fundiciones especiales con elevados contenidos de silicio $(3,5-4,5 \%)$ y/o níquel $(18-38 \%)^{[8-10]}$, tratadas con $\mathrm{FeSiMg}$, el GCH prácticamente no constituye un problema en la fabricación de piezas con módulos de enfriamiento inferiores a $2,5 \mathrm{~cm}^{[4]}$. En el caso de las piezas de mayor tamaño, existen remedios experimentales los cuales se usan de forma convencional para evitar la aparición de este defecto (utilización de enfriadores, adición de pequeñas cantidades de antimonio, etc.). Sin embargo, estas prácticas pueden derivar en otras malformaciones grafíticas o perjudicar la obtención de estructuras completamente ferríticas. Por otra parte, las exigencias del mercado se orientan a la fabricación de piezas para el sector eólico con secciones más grandes y módulos de enfriamiento cercanos o superiores a $10 \mathrm{~cm}$. En estos casos, los requerimientos de resistencia al impacto y comportamiento a fatiga también son más exigentes dada la creciente demanda de energía procedente de este sector.

La obtención de muestras de laboratorio que contengan GCH y permitan estudiar su formación es complicada. En estos casos, es necesario modificar la composición química de la aleación utilizada y/o colar muestras cuya velocidad de enfriamiento sea lo suficientemente lenta como para favorecer la formación y crecimiento del defecto. En estas condiciones, las muestras obtenidas son de elevado peso, lo que dificulta tanto su preparación como la posibilidad de obtener un número elevado de ellas, o requieren adiciones de elementos no vinculados necesariamente a la fabricación de las piezas en condiciones industriales.

Trabajos publicados anteriormente $\mathrm{e}^{[1,4,5 \text { y } 11-14]}$ han permitido estudiar las diferentes etapas del proceso de solidificación del metal en piezas fundidas con pesos que oscilan entre 60 y $200 \mathrm{~kg}$. De este modo, ha sido posible determinar las principales variables de proceso que afectan a la formación del GCH. Cuando se emplean cargas con bajos contenidos de elementos de aleación, únicamente se detecta $\mathrm{GCH}$ cuando la velocidad de solidificación es lenta. En estas condiciones, se ha comprobado que la utilización de altos contenidos de silicio y/o un exceso de inoculación en el molde incrementa considerablemente la formación de esta malformación grafítica ${ }^{[4]}$. Por otra parte, se ha observado que la presencia de ciertos contenidos de cerio $(<0,015 \%)$, adicionados deliberadamente o procedentes de la aleación nodulizante y/o el inoculante, también tiene una influencia relevante en la formación del $\mathrm{GCH}^{[12-14]}$.

La minimización del tamaño de las muestras sometidas a estudio simplifica su fabricación e incrementa las posibilidades para modificar variables experimen- tales. En la bibliografía existen trabajos en los que se obtiene GCH en piezas de espesor delgado, bien mediante la presencia de elevados contenidos de níquel en el metal ${ }^{[8 \text { y } 9]} \mathrm{O}$ introduciendo éstas en hornos especiales con el fin de reducir la velocidad de solidificación y aproximarla a las condiciones de enfriamiento en piezas de elevado espesor ${ }^{[15-17]}$. En el presente trabajo, se aplica el conocimiento previamente adquirido sobre la formación del defecto a la fabricación de muestras con secciones inferiores a $50 \mathrm{~mm}$. Las diferentes condiciones experimentales empleadas permiten evaluar la influencia de las variables de proceso en la cantidad de GCH obtenida en este tipo de secciones. Adicionalmente, se ha intentado relacionar la incidencia del GCH con el contenido de oxígeno en el metal de colada, ya que este elemento juega un papel importante en la morfología grafítica obtenida ${ }^{[18}$ y 19$]$.

\section{EXPERIMENTAL}

Todas las aleaciones fundidas se han preparado en un horno de inducción de media frecuencia $(250 \mathrm{~Hz})$, con $100 \mathrm{~kW}$ de potencia y capacidad para $100 \mathrm{~kg}$. Las cargas metálicas estaban constituidas por el $50 \%$ de lingote y el $50 \%$ de retornos procedentes de la fabricación de piezas ferríticas pertenecientes al sector eólico. Los contenidos de carbono y silicio se han variado deliberadamente mediante la adición de grafito de electrodo (\% en peso, $\mathrm{C}=98,6$ ) y de $\mathrm{FeSi}$ (\% en peso, $\mathrm{Si}=75,1 ; \mathrm{Ca}=0,3 ; \mathrm{Al}=0,8$ y Fe $=23,8$ ), respectivamente, con la finalidad de obtener aleaciones base hipoeutécticas, cercanas al eutéctico e hipereutécticas.

Los tratamientos de esferoidización del metal base se realizaron adicionando $0-0,14 \mathrm{~kg}(0-0,28 \%)$ de Cerio Mischmetal (CeMM) en forma de briquetas de $50 \mathrm{~g}$ (\% en peso, $\mathrm{Ce}=66,7 ; \mathrm{La}=33,0 ; \mathrm{Fe}=0,3$ ) y/o $0-0,8 \mathrm{~kg}(0-1,6 \%)$ de FeSiMg (\% en peso, $\mathrm{Si}=43,5 ; \mathrm{Mg}=6,0 ; \mathrm{Ca}=1,0 ; \mathrm{Al}=0,5 ;$ Tierras Raras $=1,1 ; \mathrm{Fe}=47,9$ y granulometría $1-10 \mathrm{~mm}$ ) según la metodología sándwich. Antes del vertido del metal base desde el horno de fusión, el producto nodulizante se colocó en el interior de la cámara de reacción situada en el fondo de una cuchara con capacidad para $50 \mathrm{~kg}$. Como material cubriente se utilizaron recortes de acero (tamaño $5-15 \mathrm{~mm}$ ) obtenidos a partir de procesos de estampación. Tras finalizar los tratamientos, el metal se desescorió convenientemente. En 7 de las cucharas preparadas, se tomaron muestras con el fin de registrar y analizar las curvas de solidificación utilizando tazas estándar y el sistema Thermolan ${ }^{\circledR}{ }^{[19]}$. Previamente a la colada de las tazas, se adicionó el 0,20 \% de un ino- 
culante comercial ( $\%$ en peso, $\mathrm{Si}=74,4 ; \mathrm{Al}=4,1$; $\mathrm{Ca}=1,2$; Tierras Raras $=0,5 ; \mathrm{Fe}=19,8$ y granulometría 0,2 - 0,6 mm) en el fondo de éstas. En estas 7 cucharas, también se registró la curva de solidificación correspondiente al metal colado en los moldes empleados para fabricar las cuñas, colocando un termopar tipo K aproximadamente en el centro geométrico de la cavidad contenida en los moldes. El registro de los datos de temperatura se efectuó utilizando un software diseñado expresamente para este propósito. El análisis posterior de las curvas obtenidas se efectuó siguiendo una metodología análoga a los registros procedentes de las tazas estándar ${ }^{[19]}$.

Las 15 piezas fabricadas y analizadas en el presente estudio son cuñas estándar tipo II (Fig. 1 (a)), de acuerdo a la norma UNE-EN 1563. Los moldes utilizados para fabricar estas cuñas estaban constituidos por mezclas de arena aglomerada químicamente. Estos moldes contenían tres cavidades o huellas, cada una de las cuales configuraba una cuña (Fig.1 (b)). La inoculación del metal de colada se realizó colocando el 0,45 \% de un inoculante comercial (\% en peso, $\mathrm{Si}=75,4$; $\mathrm{Al}=4,2 ; \mathrm{Ca}=0,9 ;$ Tierras Raras $=0,5 ; \mathrm{Fe}=19,0$ y morfología en forma de lingotes) en la bajante de llenado de cada molde y justo antes de la colocación del filtro (Fig. 1 (b)). Adicionalmente, en la parte inferior de cada huella se añadió el 0,20 \% del producto inoculante indicado anteriormente, pero en este caso con una granulometría de 0,2-0,6 mm.
Tras completar su proceso de enfriamiento, las 15 cuñas obtenidas a partir de la huella central se desmoldearon, y, posteriormente, se cortaron hasta obtener una sección transversal de $15 \mathrm{~mm}$ de espesor a partir de su zona central (Fig. 2 (a)). En la mayoría de las ocasiones, es posible evaluar la superficie afectada por el GCH en dicha sección transversal debido a su tonalidad más oscura (Fig. 2 (b)). En todos los casos, el estudio metalográfico posterior permitió definir correctamente la superficie afectada por la degeneración grafítica $\left(\mathrm{C}_{\mathrm{C}}\right)$. Por otra parte, la intensidad de $\mathrm{GCH}$ en el interior de las superficies afectadas por el defecto se define con ayuda del parámetro $A_{A}{ }^{[4]}$. De este modo, es posible definir el porcentaje de área total afectada por el $\mathrm{GCH}\left(\mathrm{S}_{\mathrm{S}}\right)$ a través de la siguiente ecuación:

$$
S_{S}(\%)=\left(C_{C} \times A_{A}\right) / 100
$$

En 6 de estas cuñas, se realizaron análisis de composición química en un área con gran intensidad de $\mathrm{GCH}$ (elevado valor de $\mathrm{A}_{\mathrm{A}}$ ) y en otro área con grafitos perfectamente nodulares $\left(A_{A}=0\right)$. En las 9 piezas restantes, se determinó la composición química únicamente en la zona central de la sección transversal. La determinación de los contenidos de carbono, azufre y oxígeno se efectuó mediante técnicas de análisis por combustión (Leco CS-244 y Leco Tc-436). Los contenidos de silicio se determinaron mediante
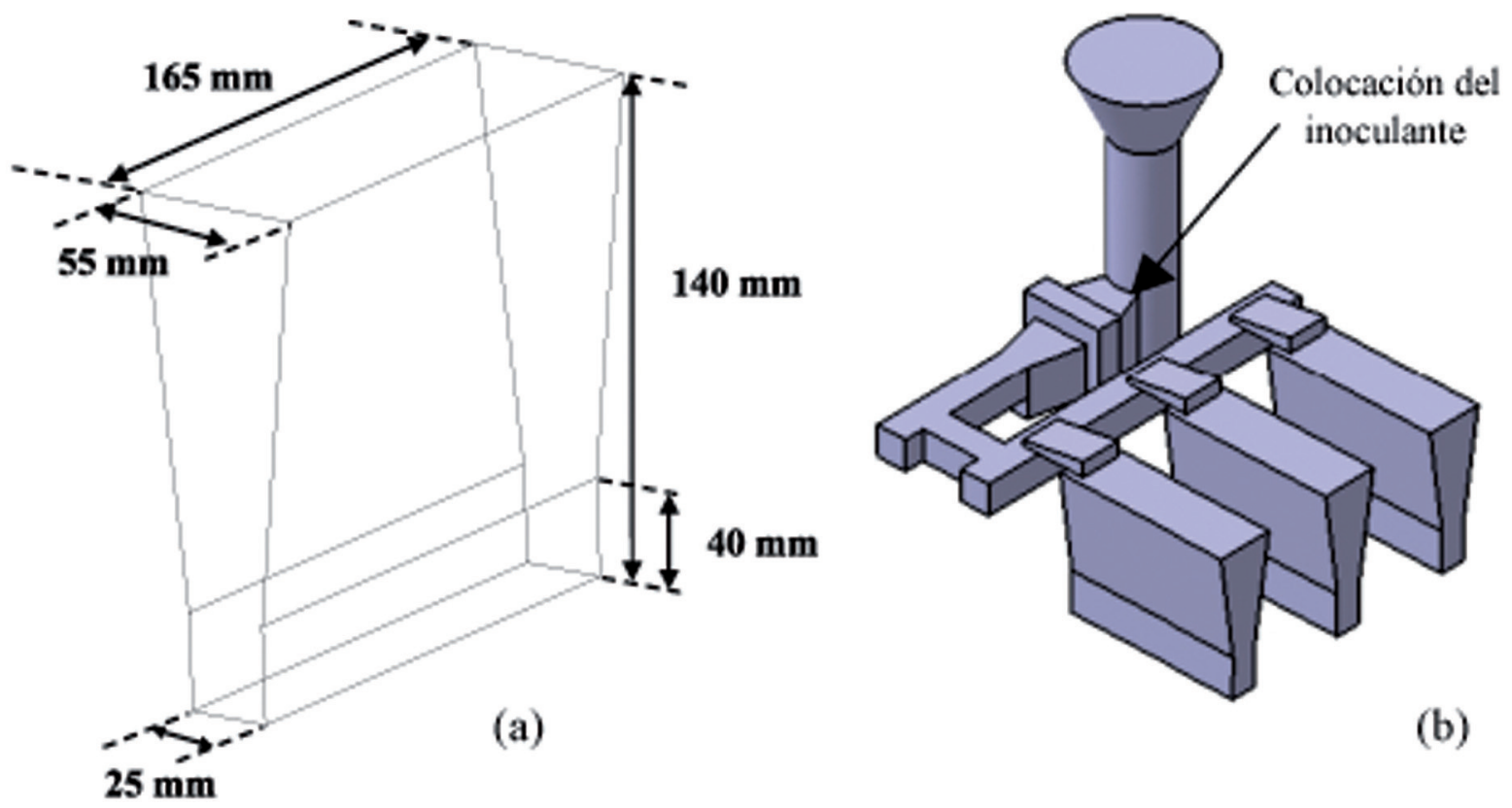

$25 \mathrm{~mm}$

Figura 1. a) Esquema de las cuñas fabricadas; b) Disposición de las mismas en el molde.

Figure 1. a) Drawing of the keel-blocks; b) Layout in the mould. 

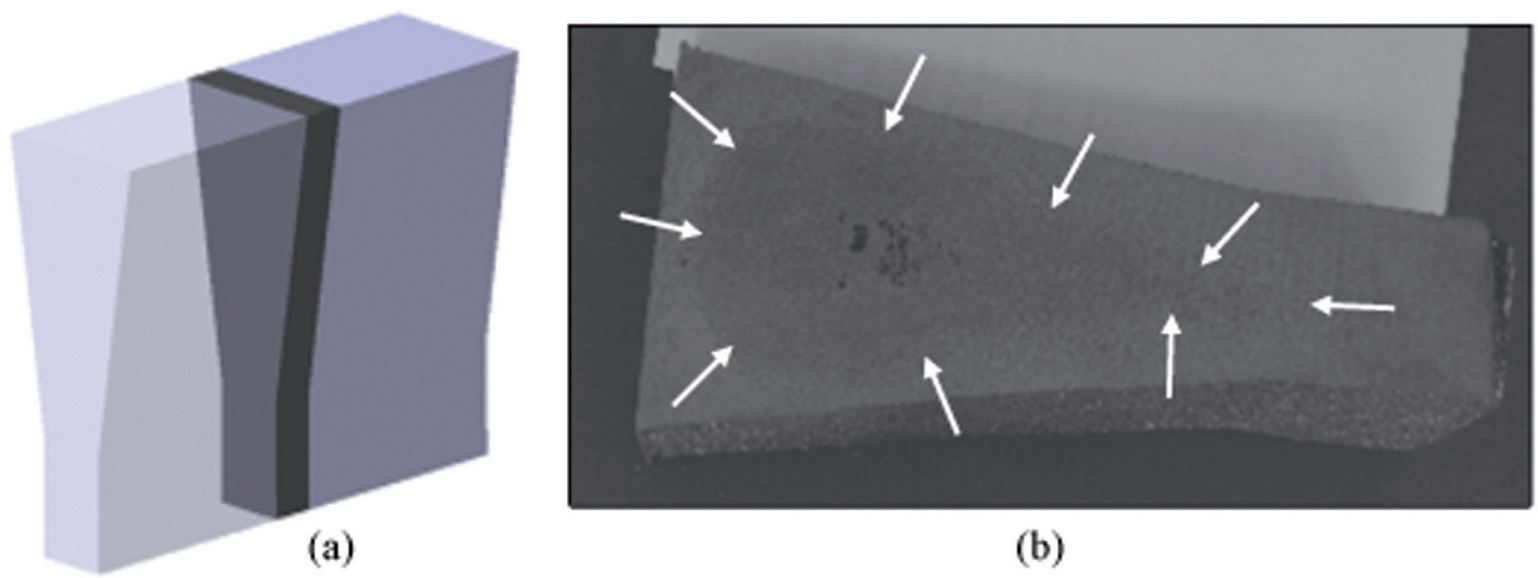

(b)

Figura 2. a) Sección transversal de la cuña; b) La tonalidad más oscura corresponde al área afectada por el grafito chunky.

\section{Figure 2. a) Transversal section of the keel-block; b) The darker zone corresponds to chunky graphite.}

gravimetría y, para el resto de elementos, se utilizó la espectrometría de emisión con plasma acoplado inductivamente (ICP-OES Perkin Elmer Optima 5300 DV) y la espectrometría de masas (ICP-MS Agilent $7500 \mathrm{ce}$ ). Los contenidos de oxígeno mostrados corresponden al promedio obtenido en cinco réplicas analizadas sobre la misma muestra. Cabe destacar que la variabilidad en este parámetro es $8 \mathrm{ppm}$ para concentraciones de oxígeno entre 25 y $50 \mathrm{ppm}$ y $5 \mathrm{ppm}$ para contenidos inferiores a $25 \mathrm{ppm}$.

\section{RESULTADOS Y DISCUSIÓN}

La tabla I muestra los valores obtenidos para los parámetros $\mathrm{S}_{\mathrm{S}}, \mathrm{C}_{\mathrm{C}}$ y $\mathrm{A}_{\mathrm{A}}$ en todas las cuñas fabricadas, así como el producto nodulizante empleado. En la tabla I se incluyen también todos los resultados obtenidos a partir de los análisis químicos efectuados sobre las cuñas fabricadas. Los contenidos de los elementos se expresan en porcentaje en peso para el carbono, silicio, carbono equivalente (CE) y manganeso, y en partes por millón en peso (ppm) para el resto de elementos.

$\mathrm{Al}$ analizar los valores de $\mathrm{S}_{\mathrm{S}}, \mathrm{C}_{\mathrm{C}}$ y $\mathrm{A}_{\mathrm{A}}$ mostrados en la tabla I se observa que la formación de $\mathrm{GCH}$ afecta a la gran mayoría de las cuñas analizadas. Este resultado es consecuencia de la aplicación de los factores mencionados anteriormente y que promueven la formación del defecto durante el proceso de fabricación de las piezas. Dado que la velocidad de enfriamiento en la zona central de las cuñas es relativamente elevada (aproximadamente $100 \mathrm{~K} / \mathrm{min}$ en el período líquido previo al inicio de la solidificación), el exceso de inoculación, los elevados contenidos de silicio y, especialmente, los niveles más altos de cerio incrementan el área afectada por este tipo de malformación grafítica. El hecho de que las cuñas no 9 y 14 no presenten $\mathrm{GCH}$ está directamente relacionado con los bajos contenidos de cerio en estas piezas (51 y 47 ppm) debido al empleo del FeSiMg como único producto esferoidizante. En la cuña no 15 , a pesar de que la cantidad de cerio es también reducida por la misma causa, sí aparece el defecto debido a la elevada concentración de silicio en el metal (3,96 \%). Cuando se emplean conjuntamente los productos FeSiMg y CeMM para llevar a cabo el tratamiento de nodulización (cuña no 3 ) también se obtiene $\mathrm{GCH}$.

Estos resultados muestran una gran contribución del cerio en la formación y posterior crecimiento del GCH durante el proceso de solidificación. Sin embargo, no ha sido posible establecer una relación clara entre el contenido de este elemento y los parámetros $\mathrm{C}_{\mathrm{C}}, \mathrm{A}_{\mathrm{A}}$ o $\mathrm{S}_{\mathrm{S}}$ (Fig. 3), probablemente debido a la importante influencia de los otros factores que contribuyen a la formación de esta degeneración grafítica.

Cuando se comparan los valores de los parámetros $\mathrm{C}_{\mathrm{C}}$ y $\mathrm{A}_{\mathrm{A}}$, obtenidos en las cuñas tratadas únicamente con el producto CeMM, se observa una relación directa entre ellos (Fig. 4). Es decir, la superficie afectada por el GCH $\left(\mathrm{C}_{\mathrm{C}}\right)$ aumenta con su intensidad $\left(\mathrm{A}_{\mathrm{A}}\right)$. Esta relación es contraria a la encontrada en trabajos previos $^{[4,11-14]}$, realizados sobre bloques cúbicos de fundición esferoidal con elevado módulo de enfriamiento y tratados exclusivamente con FeSiMg. En estas pie- 


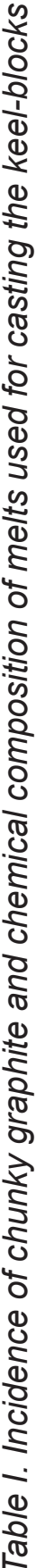

U

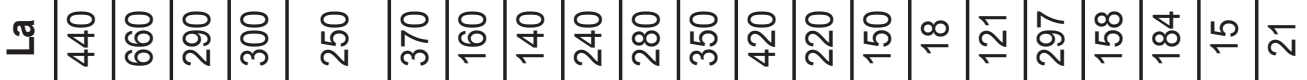
क 음ऽ

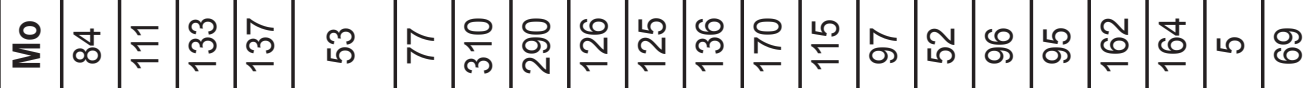
ব্ত 요

¿

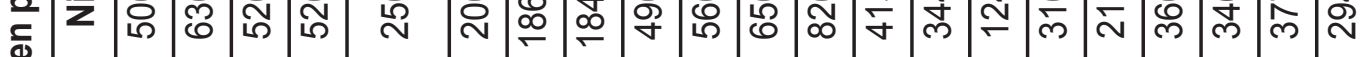

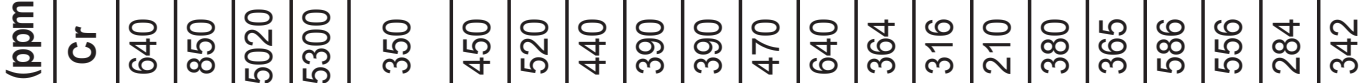

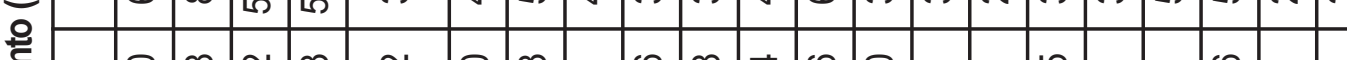

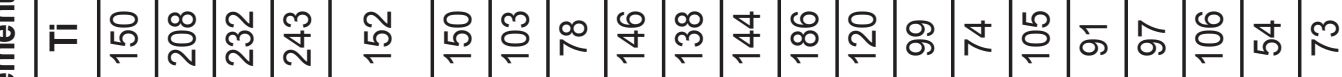
《舟

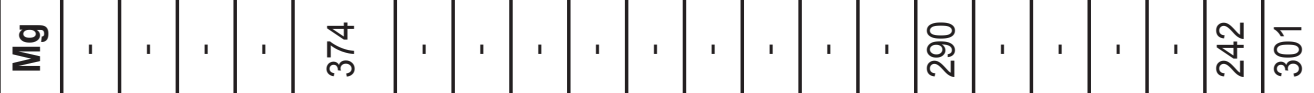

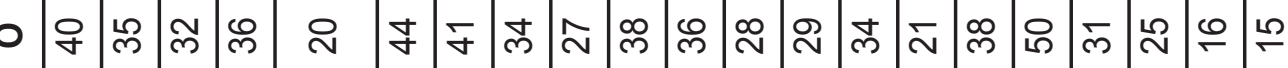

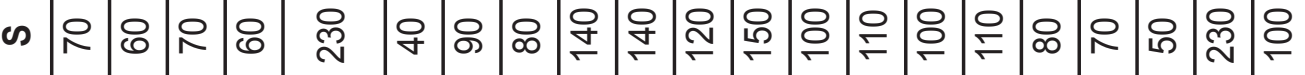

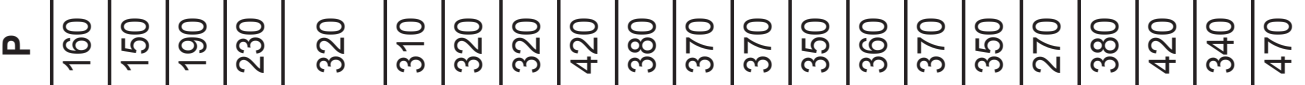

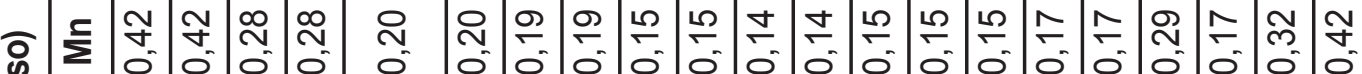

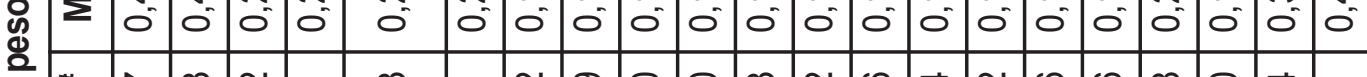
๘ $\therefore 0$ ○

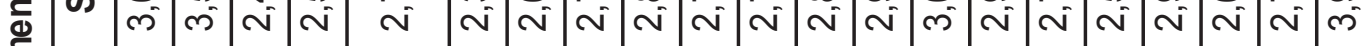

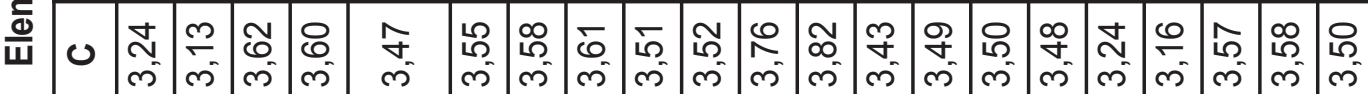

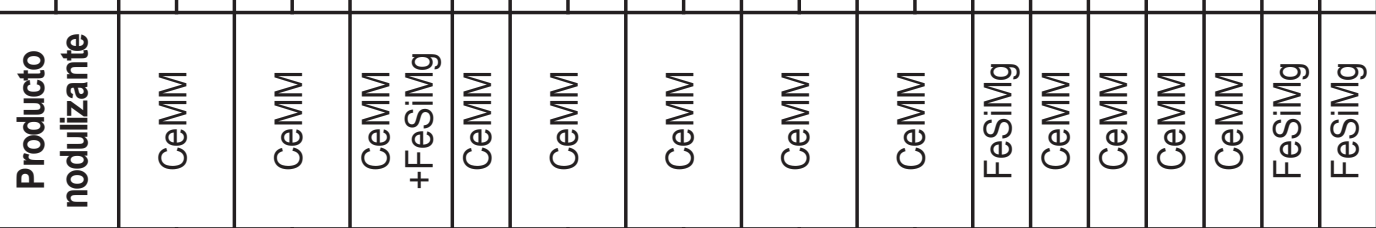

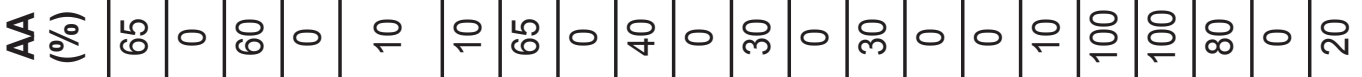

\begin{tabular}{|c|c|c|c|c|c|c|c|c|c|c|c|c|c|c|c|}
\hline Uః & $\stackrel{N}{N}$ & $\stackrel{5}{7}$ & 우 & 음 & $\infty$ & $\stackrel{\Delta}{\sim}$ & న & D & 0 & $\stackrel{2}{2}$ & $\infty$ & $\infty$ & $\infty$ & 0 & ת \\
\hline $\mathscr{\infty} \cong$ & 守 & $\widehat{\sim}$ & - & - & กิ & 으 & $\sigma$ & 0 & 0 & $\sim$ & $\stackrel{\infty}{\infty}$ & $\stackrel{\infty}{\infty}$ & छ & 0 & $\checkmark$ \\
\hline ؟ & $\leftarrow$ & $\sim$ & $m$ & $\stackrel{*}{\forall}^{\prime}$ & ما & 0 & & $\infty$ & as & 은 & $\mp$ & $\cong$ & $m$ & $\stackrel{\nabla}{ }$ & 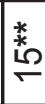 \\
\hline
\end{tabular}




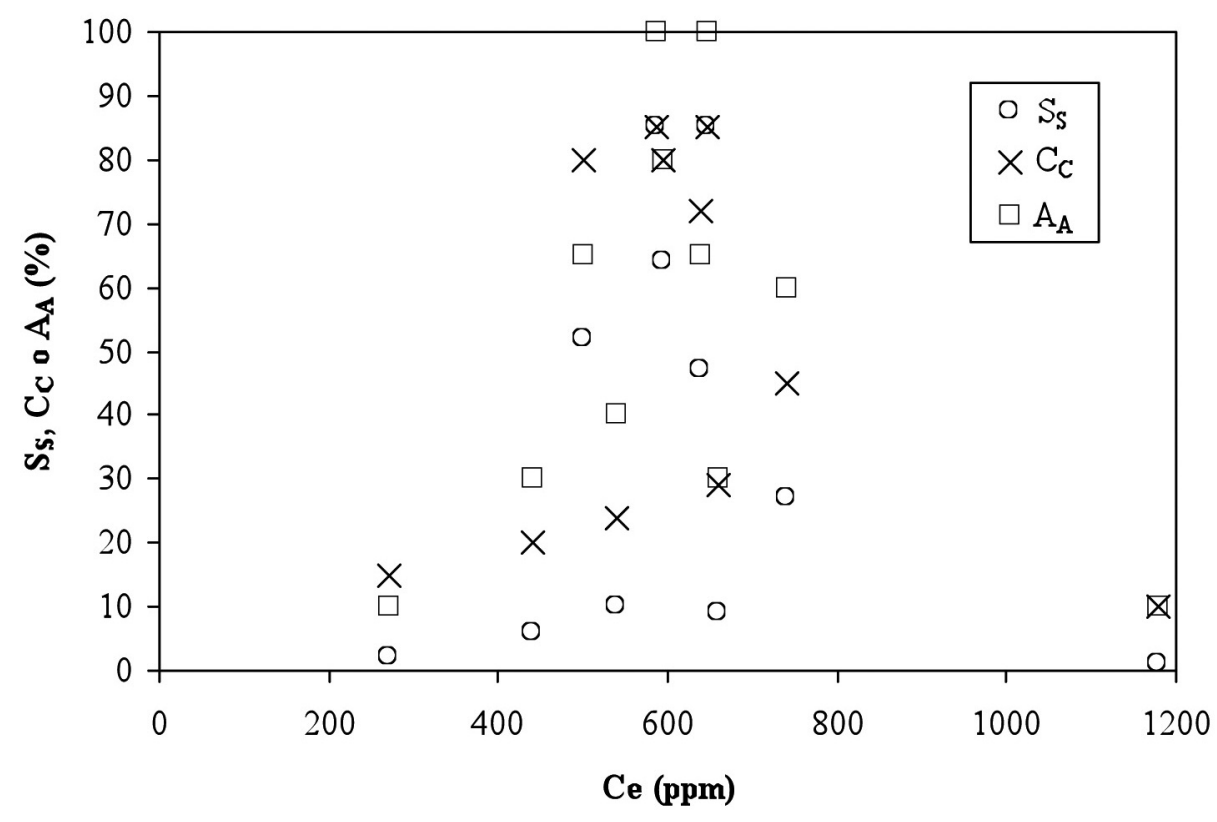

Figura 3. Relación de los parámetros $S_{S}, C_{C}$ y $A_{A}$ con el contenido de Ce en el metal.

\section{Figure 3. Correlation between $S_{S}, C_{C}, A_{A}$ parameters and Ce content in the alloy.}

zas de gran tamaño, cuando aumenta el volumen afectado por el GCH disminuye considerablemente la intensidad del mismo. La causa de este comportamiento opuesto debe estar vinculada al cambio en el elemento esferoidizante empleado (Ce en lugar de $\mathrm{Mg}$ ) y/o a la gran diferencia entre las velocidades de enfriamiento de las cuñas (módulo $1,2 \mathrm{~cm}$ ) y los bloques cúbicos (módulo $5 \mathrm{~cm}$ ). El hecho de que la única cuña tratada exclusivamente con $\mathrm{FeSiMg}$ y que contiene $\mathrm{GCH}\left(\mathrm{n}^{\circ} 15\right)$ muestre el mismo tipo de relación $\mathrm{C}_{\mathrm{C}}$ vs. $\mathrm{A}_{\mathrm{A}}$ que la observada para los bloques cúbicos de elevado módulo parece indicar que la causa puede ser el tipo de agente esferoidizante utilizado.

El análisis comparativo de las curvas de solidificación registradas en el presente trabajo muestra la capacidad del cerio para reducir los valores de la temperatura eutéctica mínima $\left(\mathrm{Te}_{\text {mín }}\right)$ y aumentar el subenfriamiento, este último calculado como $\Delta \mathrm{T}=1154+(4 \times \% \mathrm{Si})-\mathrm{Te}_{\text {mín }}$. La figura 5 muestra la evolución de estos dos parámetros con el contenido de cerio en el metal de colada. En aquellos casos en los que se dispone de dos valores de concentración de silicio y cerio por cuña, se han seleccionado los valores correspondientes a la zona afectada por el GCH (área central donde se localiza el termopar). El caso más extremo viene dado por la cuña no 4 (Tabla I), donde el contenido de cerio alcanza los 1.180 ppm, la $T e_{\text {mín }}$ disminuye a valores propios de una fundición con carburos (aspecto confirmado posteriormente en las inspecciones metalográficas) y el suben- friamiento es el más elevado $\left(40,3^{\circ} \mathrm{C}\right)$. Al comparar la evolución obtenida para $\mathrm{Te}_{\text {mín }} \mathrm{y} \Delta \mathrm{T}$ entre cuñas y tazas, se comprueba cómo la velocidad de enfriamiento contribuye al efecto provocado por el aumento del contenido de cerio. Es decir, en las tazas (mayor velocidad de enfriamiento) la $\mathrm{Te}_{\text {mín }}$ disminuye más rápidamente a bajos contenidos de cerio (línea continua de trazo fino) y el $\Delta \mathrm{T}$ aumenta en condiciones similares (línea discontinua de trazo fino). En el caso de las cuñas, el comportamiento es el contrario, la variación más crítica en ambos parámetros se obtiene cuando la concentración de cerio en el metal es superior a $600 \mathrm{ppm}$.

No debe considerarse la existencia de una relación entre el aumento del área afectada por el GCH y valores más elevados del parámetro $\Delta \mathrm{T}$. Un ejemplo de este hecho son los resultados obtenidos para las cuñas no 3 y 10 (Tabla I). En ambos casos, el metal presenta contenidos similares de carbono y silicio. Sin embargo, el área con defecto es comparable o incluso ligeramente mayor en la cuña $\mathrm{n}^{\circ} 10$, donde los contenidos de antimonio y cerio son 11 veces superior y 2,8 veces inferior, respectivamente, y el subenfriamiento es menor $\left(11,3^{\circ} \mathrm{C}\right)$ que en la cuña $\mathrm{n}^{\circ} 3\left(17,3^{\circ} \mathrm{C}\right)$. En este caso, la presencia de magnesio en el metal utilizado para fabricar la cuña $\mathrm{n}^{-} 3$ parece ser un factor determinante a la hora de compensar el efecto del cerio y reducir la cantidad de defecto obtenida. Este comportamiento coincide con los resultados obtenidos en trabajos publicados ante- 


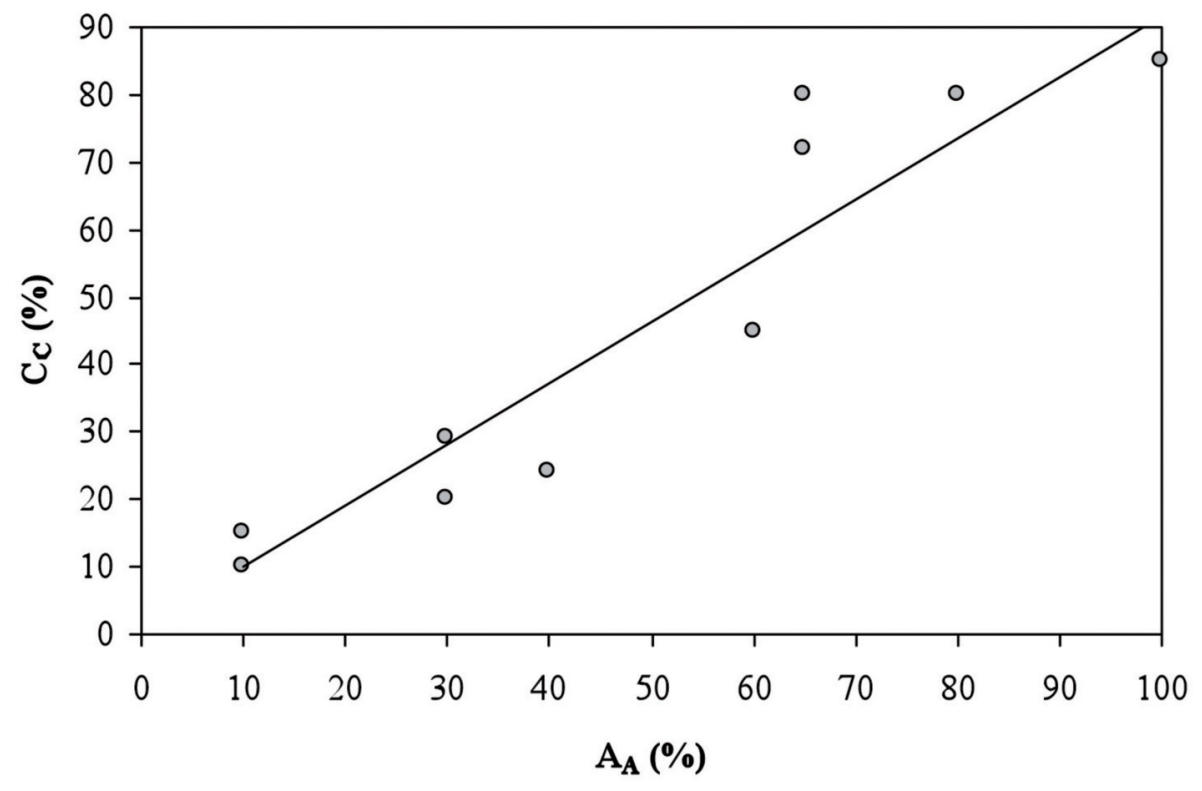

Figura 4. Relación entre los parámetros $C_{C}$ y $A_{A}$ en las aleaciones tratadas únicamente con CeMM.

Figure 4. Correlation between $C_{C}$ and $A_{A}$ parameters in alloys treated only with CeMM.

riormente ${ }^{[14]}$. En otro caso descrito en la bibliografía ${ }^{[4]}$, se observa reiteradamente la obtención de mayores cantidades de $\mathrm{GCH}$ en metales inoculados y con valores de $\Delta \mathrm{T}$ más bajos en relación a los correspondientes metales no inoculados.
Por otra parte, los valores de $\mathrm{Te}_{\text {mín }}$ obtenidos a partir de las curvas correspondientes a las dos cuñas tratadas únicamente con $\mathrm{FeSiMg}\left(1.158,2^{\circ} \mathrm{C}\right.$ para la cuña $\mathrm{n}^{\circ} 14$ y $1.157,9^{\circ} \mathrm{C}$ para la $\mathrm{n}^{\circ} 15$ ) son similares o, en muchos casos, incluso superiores a los

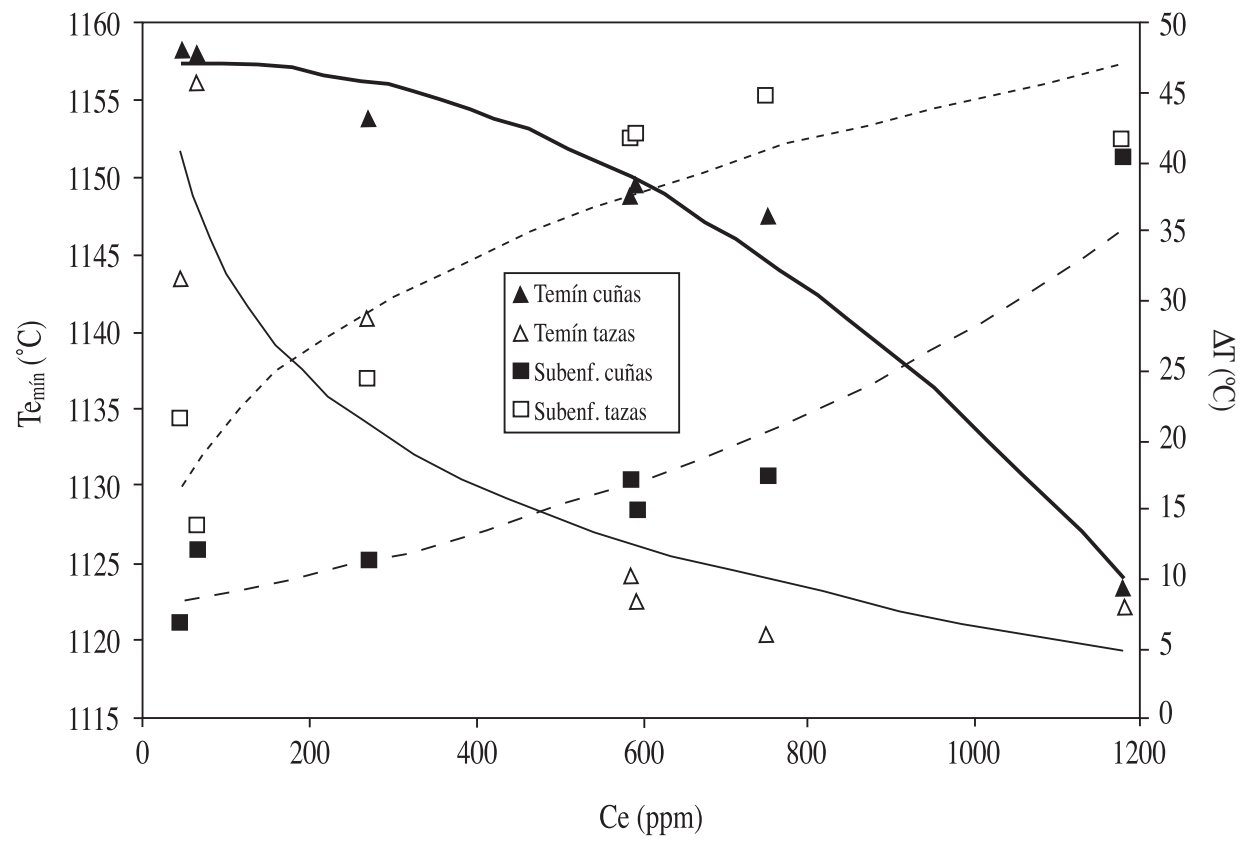

Figura 5. Evolución de la $\mathrm{Te}_{\text {mín }}$ y $\Delta \mathrm{T}$ en función del contenido de Ce.

Figure 5. $T e_{\min }$ and $\Delta T$ evolution related to Ce content. 
alcanzados en los bloques cúbicos de elevado espe$\operatorname{sor}^{[11 \text { y } 12]}\left(1.147-1.161^{\circ} \mathrm{C}\right)$. Este hecho tiene lugar a pesar de la gran diferencia de módulos existente entre ambos tipos de pieza y puede ser atribuido a los altos contenidos de silicio en el metal empleado para fabricar dichas cuñas $(\mathrm{Si}=2,73$ y 3,96 \%, incluyendo la sobreinoculación realizada en los moldes) con respecto a los bloques cúbicos $(\mathrm{Si}=1,90-2,40 \%)$. Así mismo, los valores de $\Delta \mathrm{T}$ pueden considerarse comparables en piezas con bajos contenidos de cerio $\left(6,7-11,9^{\circ} \mathrm{C}\right.$ en las cuñas y $4,2-10,6^{\circ} \mathrm{C}$ en los bloques cúbicos ${ }^{[11}$ y 12] $)$ y afectadas de forma muy diferente por la presencia del GCH. Por tanto, el aumento en la cantidad de este defecto parece estar vinculado a la presencia de cerio en el metal y no al subenfriamiento obtenido durante la solidificación. Este aspecto parece contradecir determinados resultados publicados anteriormente ${ }^{[9 \text { y } 20]}$, donde se relaciona la mayor incidencia del GCH con reducciones en el subenfriamiento de fundiciones esferoidales con importantes contenidos de níquel. En estos casos, la causa que permite incrementar la formación del GCH es la disminución de la velocidad de enfriamiento del material y, como consecuencia de esto último, se obtienen valores del subenfriamiento más bajos.

En general, las composiciones químicas obtenidas a partir de las zonas afectadas por el $\mathrm{GCH}$ y aquellas que contienen únicamente grafitos correctamente esferoidales son similares (Tabla I). Estos resultados ponen de manifiesto tanto la ausencia de fenómenos

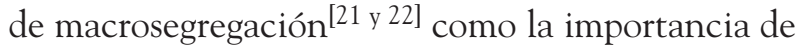
todos los factores que promueven la formación del $\mathrm{GCH}$, incluyendo la velocidad de enfriamiento local en cada una de las zonas de la pieza. En relación a la presencia de oxígeno en el metal, a pesar del potente efecto desoxidante del cerio ${ }^{[21]}$, se observa que aquellas aleaciones tratadas con CeMM presentan contenidos de oxígeno sistemáticamente mayores (25 - $50 \mathrm{ppm}$ ) que las tratadas con FeSiMg (15 - 21 ppm). Este aspecto puede guardar relación con la intensidad de la reacción provocada durante los tratamientos de esferoidización. El CeMM no origina reacciones violentas con proyecciones del metal líquido fuera de la cuchara, tal y como ocurre cuando se emplea el FeSiMg. De este modo, la cantidad de escoria obtenida en el metal tratado con la ferroaleación que contiene magnesio es mayor, lo que puede explicar los menores niveles de oxígeno presentes en este tipo de aleaciones.

La figura 6 muestra un análisis comparativo de los contenidos de oxígeno obtenidos a partir de un grupo de fundiciones de hierro con diferentes morfologías grafíticas, además de las aleaciones fabricadas en el presente trabajo (en aquellos casos en los que se dispone de dos contenidos de este elemento para una misma muestra, se considera el valor medio). En todos estos materiales, la metodología de análi-

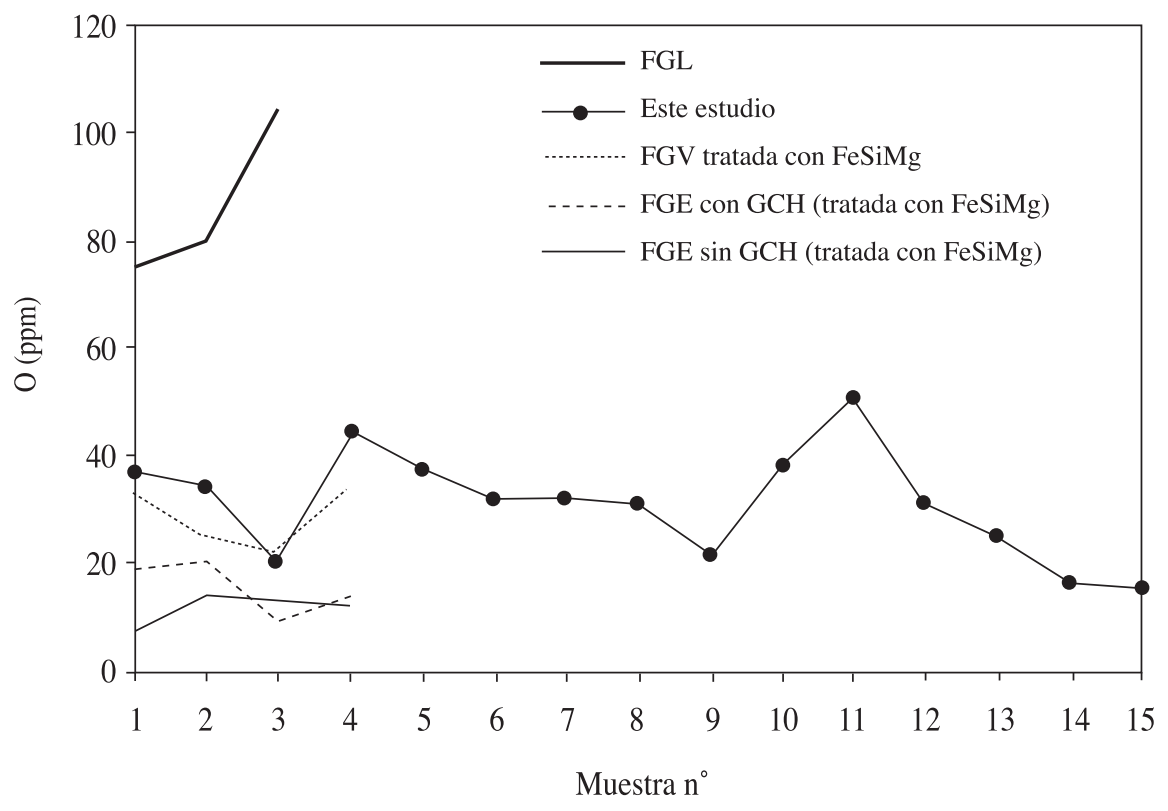

Figura 6. Estudio comparativo del contenido de oxígeno para distintos tipos de fundición de hierro.

Figure 6. Comparative study of the oxygen content in different cast iron types. 
sis utilizada es la indicada en la sección experimental. Los niveles de oxígeno en las aleaciones tratadas con CeMM son similares o incluso ligeramente superiores a los obtenidos a partir de las fundiciones con grafito vermicular (FGV), claramente inferiores al caso de las fundiciones laminares (FGL) y superiores a los pertenecientes a las fundiciones que contienen grafito esferoidal (FGE) tratadas con FeSiMg. Los 4 materiales fabricados en este trabajo y tratados con FeSiMg (cuñas no 3, 9, 14 y 15) muestran contenidos de oxígeno similares a los obtenidos en las fundiciones esferoidales tratadas con este tipo de producto nodulizante y que han sido seleccionadas con fines comparativos.

De acuerdo con la comparativa mostrada en la figura 6, es posible obtener $\mathrm{GCH}$ con diferentes composiciones químicas y contenidos de oxígeno en el metal. Por ello, no parece posible establecer una dependencia de esta malformación grafítica, basada en el grado de desoxidación de la aleación. En este sentido, determinadas publicaciones ${ }^{[9,20,21}$ y 23] relacionan las condiciones de formación del defecto con diferentes niveles de oxígeno en el metal, estableciendo vínculos con la capacidad de nucleación grafítica $^{[9}$ y 21] o incluso llegando a situar este tipo de morfología grafítica en un campo intermedio entre la nodular y la laminar ${ }^{[23]}$. Sin embargo, en la actualidad son numerosas las evidencias que no permiten efectuar este tipo de asignaciones. En el presente trabajo, la utilización de cerio en la fabricación de los materiales analizados ha mostrado tanto su capacidad para originar grafitos esferoidales en ausencia de magnesio, como su potente efecto promotor del GCH. Por otra parte, también ha sido posible obtener este defecto cuando la concentración de cerio en el metal de colada es reducida, utilizando determinados contenidos de magnesio $(242-301 \mathrm{ppm})$ y con niveles de oxígeno muy inferiores al detectado en las aleaciones tratadas con CeMM.

\section{CONCLUSIONES}

Las conclusiones obtenidas a partir del presente estudio son las siguientes:

- La combinación de elevados contenidos de cerio y/o silicio junto con la sobreinoculación del metal permiten obtener de forma efectiva grafito chunky en piezas sometidas a velocidades de enfriamiento relativamente altas (módulos de enfriamiento $<1,5 \mathrm{~cm}$ ).

- El cerio ha mostrado ser un elemento con capacidad para formar grafitos esferoidales, pero también es un potente promotor de grafito chunky. Los resultados obtenidos en este tra- bajo indican que la presencia de cerio en el metal es el factor de mayor influencia en la aparición de este tipo de malformación grafítica, mostrando una relación entre los parámetros $\mathrm{C}_{\mathrm{C}}$ y $\mathrm{A}_{\mathrm{A}}$ diferente a la observada en metales tratados exclusivamente con magnesio.

- El aumento de los contenidos de cerio en el metal origina disminuciones en los valores de la $\mathrm{Te}_{\text {mín }} \mathrm{y}$ aumentos importantes en el subenfriamiento. Cuando el contenido de cerio es especialmente elevado aparecen carburos en las piezas. El incremento de la velocidad de enfriamiento potencia estos efectos.

- Cuando se utiliza magnesio como elemento esferoidizante, es necesario elevar el contenido de silicio hasta valores cercanos al $4 \%$ para obtener grafito chunky en las cuñas. Este resultado es similar a los publicados anteriormente y obtenidos sobre bloques cúbicos de elevado espesor.

- No se han encontrado diferencias significativas entre las composiciones químicas de las zonas afectadas por el grafito chunky y aquellas libres de este defecto dentro de una misma pieza. Este hecho indica la ausencia de fenómenos de macrosegregación durante el enfriamiento de los materiales analizados.

- La adición de cerio al metal origina aleaciones con niveles de oxígeno similares a los encontrados en las fundiciones de hierro con grafito vermicular. Aunque se han detectado contenidos de oxígeno mayores en las piezas tratadas con CeMM que en las tratadas con $\mathrm{FeSiMg}$, no se ha encontrado relación alguna entre la formación de grafito chunky con este parámetro.

\section{Agradecimientos}

Los autores de este trabajo agradecen la ayuda prestada en la ejecución de las pruebas experimentales a los responsables de TQC Technologies, S.L.U. Este trabajo está financiado por el Departamento de Industria del Gobierno de España (PROFIT FIT030000-2007-94).

\section{REFERENCIAS}

[1] S. I. Karsay, AFS Trans. 78 (1970) 85-92.

[2] A. Javaid y C.R. Loper, AFS Trans. 103 (1995) $135-150$.

[3] R. Kallböm, Mat. Sci. Eng. A 413-414 (2005) 346-351. 
[4] I. Asenjo, P. Larrañaga, J. Sertucha, R. Suárez, J. M. Gómez, I. Ferrer y J. Lacaze, Int. J. Cast. Metal. Res. 20 (2007) 319-324.

[5] Z. Ignaszak, Int. J. Cast. Metal. Res. 16 (2003) 93-97.

[6] A. Suárez-Sanabria y J. Fernández-Carrasquilla, Rev. Met. Madrid 42 (2006) 18-31.

[7] H. Itofuji y H. Uchikawa, AFS Trans. 98 (1990) 429-448.

[8] S. I. Karsay, Modern Casting 58 (1970) 85-192.

[9] M. Gagné, C. Labrecque y A. Javaid, AFS Trans. 115 (2007) trabajo 07-004.

[10] H. Nakae, S. Jung y H. C. Shin, J. Mater. Sci. Technol. 24 (2008) 289-295.

[11] J. Lacaze, S. Armendariz, P. Larrañaga, I. Asenjo, J. Sertucha y R. Suárez, Mat. Sci. Forum 636-637 (2010) 523-530.

[12] J. Sertucha, R. Suárez, I. Asenjo, P. Larrañaga, J. Lacaze, I. Ferrer y S. Armendariz, ISIJ Int. 49 (2009) 220-228.

[13] P. Larrañaga, I. Asenjo, J. Sertucha, R. Suárez, I. Ferrer y J. Lacaze, Int. J. Cast. Metal. Res. 22 (2009) 192-195.

[14] P. Larrañaga, I. Asenjo, J. Sertucha, R. Suárez, I. Ferrer y J. Lacaze, Metall. Mater. Trans. A 40A (2009) 654-661.
[15] Z. Zhang, H. M. Flower y Y. Niu, Mater. Sci. Technol. 5 (1989) 657-664.

[16] K. Shoji, S. Masayuki, S. Haruyoshi y N. Kokichi, J. Japan Foundry Eng. Soc. 72 (2000) 311-316.

[17] H. Nakae, H. C. Shin, M. Hara y Y. Harada, J. Japan Foundry Eng. Soc. 75 (2003) 337 343.

[18] M. Homma y K. Hukuoka, Science Reports of the Research Institutes, Tohoku University, Senday, Japan, 1972, pp. 116-121.

[19] P. Larrañaga, J. M. Gutiérrez, A. Loizaga, J. Sertucha y R. Suárez, AFS Trans. 116 (2008) 547-561.

[20] M. Gagné y C. Labrecque, AFS Trans. 117 (2009) 561-571.

[21] R. Kallböm, K. Hamberg y L-E. Bjorkegren, Proc. 67 $7^{\text {th }}$ World Foundry Congress, Harrogate, England, 2006, trabajo 184.

[22] J. Lacaze, S. Méndez, J. Sertucha, P. Larrañaga, R. Suárez y I. Ferrer, Proc. The Carl Loper Cast Iron Symposium, Madison, EE.UU., 2009, pp. 59-68.

[23] X. Diao, Z. Ning, F. Cao, S. Ren y J. Sun, Key Eng. Mat. 457 (2011) 31-37. 